Dieses Dokument ist eine Zweitveröffentlichung (Verlagsversion) / This is a self-archiving document (published version):

Alexander Lasch

Soziale Medien in der externen Unternehmenskommunikation

Erstveröffentlichung in / First published in:

Markus Hundt/Dorota Biadala, Hrsg. Handbuch Sprache in der Wirtschaft. Berlin/Boston:

De Gruyter 2015, S. 297 - 323. ISBN 978-3-11-029621-1.

DOI: https://doi.org/10.1515/9783110296211-017

Diese Version ist verfügbar / This version is available on:

https://nbn-resolving.org/urn:nbn:de:bsz:14-qucosa2-748520 


\title{
15. Soziale Medien in der externen Unternehmenskommunikation
}

\begin{abstract}
externe Unternehmenskommunikation gelegt. scher Perspektive noch beinahe unbestellt ist.

$1 \quad$ Neue Medien und Soziale Medien in der externen und internen UK

2 Markenkommunikation im Web 2.0

3 Exemplarische Analyse

4 Fazit und Ausblick

5 Literatur
\end{abstract}

Abstract: Der Artikel setzt sich im Dialog mit der aktuellen Forschung mit der Bedeutung der Sozialen Medien für die Unternehmenskommunikation auseinander. Da sich die interne und die externe Unternehmenskommunikation strukturell nur hinsichtlich weniger Parameter unterscheiden (u.a. in den Fragen der Thematik und der Adressaten), wird in der exemplarischen Analyse das Hauptaugenmerk auf die

Im Mittelpunkt des Beitrags steht die Frage, ob und wie mittels Sozialer Medien kommunikativ anschlussfähiges Wissen durch Sprache an einem spezifischen Ort konstituiert werden kann und wer an diesem Prozess beteiligt ist. Dabei ist zu beachten, dass dieses Forschungsfeld mit dem Fokus auf (Corporate) Blogs aus linguisti-

Der Schwerpunkt der Hinführung wird nach einer Diskussion des Forschungsstandes zum Thema (Kap.1) auf der interaktiven Grundlegung der Sozialen Medien liegen (Kap. 2). Diese stellt für die Unternehmenskommunikation eine enorme Herausforderung dar. Wissen über und von einem Unternehmen, einer Marke, einem Selbstbild (Kap. 2.2) u. a. kann durch Sprache nicht mehr unter den Bedingungen der Massenkommunikation konstituiert werden, sondern muss gemeinsam mit einem Gegenüber ,im Dialog، etabliert und stabilisiert werden. Das Hauptaugenmerk einer exemplarischen Analyse (Kap. 3) wird auf einem ausgewählten ,Corporate Blog` liegen.

\section{Neue Medien und Soziale Medien in der externen und internen Unternehmenskommunikation}

\subsection{Hinführung: Die Begriffe Interaktion, Kommunikation und Marketing in der Forschung zur Unternehmenskommunikation}

Die externe und interne Unternehmenskommunikation ist ein Teilbereich der Angewandten Linguistik, in dem Forschungsergebnisse aus einzelnen linguistischen 
Teildisziplinen auf die Anforderungen der Wirtschaft treffen, Unternehmensziele strategisch $\mathrm{zu}$ planen und gegenüber Mitarbeitern des Unternehmens (intern) auf der einen und Kunden des Unternehmens (extern) auf der anderen Seite (vgl. Bruhn 2010) zu kommunizieren. Die Idee, Kommunikationsprozesse planen und steuern zu können (vgl. Bruhn 2009, 203), ist nicht nur relevant für das Marketing eines Unternehmens, das „die zielorientierte Gestaltung aller marktgerichteten Unternehmeraktivitäten“ (Esch/Herrman/Sattler 2008, 29) meint, sondern es erfasst auch die Interaktion zwischen Mitarbeitern und Kunden eines Unternehmens, die der externen Unternehmenskommunikation zuzurechnen sind (anders Bruhn 2010, 4, der hier von „interaktive[r] Kommunikation“ spricht). Auf dieser einfachen binären Unterscheidung können weitere Modelle aufruhen, die als weitere Klassifikationsmöglichkeiten die Entitäten Mitarbeiter und Kunden genauer und adäquat den kommunikativen Bedürfnissen eines Unternehmens differenzieren. Salzer $(2011,14)$ bspw. spricht bei der Unternehmenskommunikation von den Teilbereichen der Public Relations (Öffentlichkeit, Massenmedien), der Internen Kommunikation (Mitarbeiter), der Public Affairs (Meinungsführer, Politik, Behörden), der Finanzkommunikation (Investoren, Analysten, Wirtschaftsmedien) und Marktkommunikation (Vertriebspartner, Kunden, Wettbewerber) (für neuere Tendenzen vgl. Mast in diesem Band). Anders als die Forschung zur Unternehmenskommunikation wird sich dieser Artikel allerdings nicht der weiteren Ausdifferenzierung des Kommunikationsbegriffs widmen (Mast 2010, in diesem Band; Bruhn 2010; 2011; Piwinger/Zerfaß 2007; Salzer 2011). Kommunikation wird hier verstanden als verbale Interaktionsform.

Social Media wird in der Unternehmenskommunikation dem Marketing zugerechnet, wobei die Verwendung des Begriffs Marketing in der Forschung einen ähnlich dispersen Eindruck wie der der Kommunikation hinterlässt. Grundsätzlich ist darunter aber mit Salzer (2011), wie oben zitiert, „die zielorientierte Gestaltung aller marktgerichteten Unternehmeraktivitäten“ (Esch/Herrman/Sattler 2008, 29) zu verstehen. Diese ,zielorientierte Gestaltung“ kann zwar weiter differenziert werden, was im Rahmen der Unternehmenskommunikation bisher allerdings nicht systematisch erfolgte (vgl. exemplarisch die Mischklassifikation nach Adressat, Medium und Kommunikationssituation der „Kommunikationspolitik“ im „Marketingmix“ bei Aerni/Bruhn 2008, 30).

\subsection{Monolog und Dialog: Neue Medien und Soziale Medien}

Mit dem Begriff der so genannten Neuen Medien greift man gemeinhin auf ein Konzept zurück, um Angebote zu bezeichnen, die sich von den ,etablierten' Medien wie Radiound Fernsehfunk oder Printmedien dadurch unterscheiden, dass es Angebote der Onlinekommunikation sind. Davon abgesehen sind sie kommunikationstypologisch und strukturell den Bedingungen massenmedialer Kommunikation anderer Medien nicht nur ähnlich. Sie adaptieren diese vielmehr und nutzen dabei nur einen anderen 
Übertragungskanal, der einige neue paratextuelle Optionen bietet (Hypertextualität), die allerdings in Bezug auf die Modi der Konstitution von Wissen keine weitreichenden Folgen haben: Diese Angebote begreift man als Angebote des Web 1.0. Soziale Medien hingegen sind Signum des so genannten Web 2.0. Darunter versteht man Kommunikationsangebote, die über die reine Informationsvermittlung unter massenmedialen Bedingungen im Web 1.0 hinausreichen und zur stärkeren Partizipation einladen: Soziale Netzwerke, (Micro-)Blogging-Dienste, (Micro-)Chat-Dienste, NewsDienste, Sharing-Plattformen, Bookmarking-Dienste, Wikis usw. Die unter diesen Bedingungen stattfindende Kommunikation ist vollkommen anders strukturiert als die „Aussendung“ von einem Emittenten zu einem stummen, anonymen Publikum im Web 1.0: Kommunikation im Web 2.0 wird als „Dialog“ konzeptualisiert (vgl. exemplarisch dazu Siever/Schlobinski/Runkehl 2005; Schütz u. a. 2005; Androutsopoulos u. a. 2006; Dürscheid/Ziegler 2002; Moraldo 2009; Marx/Weidacher 2014; aus der Unternehmenskommunikation z. B. Bruhn 2011; Dörfel/Schulz 2011 und vor allem Kreutzer 2012).

Wenn im Folgenden von „Dialog“ oder „Gespräch“ die Rede sein wird, dann ist damit der medial schriftsprachliche kommunikative Austausch auf Plattformen des Web 2.0 mitgemeint. Mediale und konzeptionelle Aspekte von Mündlichkeit und Schriftlichkeit werden hier explizit ausgeklammert (vgl. aber dazu Dürscheid/Schneider 2015 und für die prinzipielle Nähe von Text- und Gesprächslinguistik exemplarisch die Arbeiten von Koch/Österreicher 1985 und 1994; Brinker u. a. 2000; Brinker u. a. 2001; sowie schließlich Birkner/Janich im Druck), da das Verhältnis gerade bei Kommunikation in Online-Umgebungen gleichermaßen Bezüge zu konzeptionell und medial schriftlichen wie mündlichen Kommunikationsformen aufweist (vgl. dazu exemplarisch die Konzeptualisierung von Chat-Kommunikation als „getippten Dialog“ bei Dürscheid/Brommer 2009).

Ein Dialog ist aus linguistischer Perspektive ein recht voraussetzungsreiches Konzept: $\mathrm{Zu}$ den Prämissen einer Linguistik des Dialogs gehört, dass Interaktivität und Pragmatizität (Deppermann 2011, 210-213), neben Zeitlichkeit, die in unserem Kontext in der Adaption auf geschriebene Sprache eine nachgeordnete Rolle spielt, neben anderen Faktoren wesentliche konstitutive Eigenschaften dialogisch organisierter gesprochener Sprache sind. Mit Interaktivität ist dabei aber nicht irgendein partnerorientiertes oder -relevantes (spechakttheoretisches) Handeln gemeint. Vielmehr wird damit im Hinblick auf zwischenmenschliche Kommunikation das Merkmal der Dialogizität aufgerufen. Von einem Dialog (von griech. dia = ,[hin-]durch ${ }^{\star}$ und $\operatorname{logos}=$,Wort') spricht man dann, wenn in einem beobachtbaren sprachlichen Format wie einem Gespräch wenigstens ein Sprecherwechsel (turntaking) vollzogen wurde, was wiederum voraussetzt, dass zwei nicht identische Sprecher jeweils einen Gesprächsschritt (turn) realisiert haben (vgl. Brinker/Sager 2006, 62-77). Nur auf dieser Basis können größere Gesprächseinheiten aufbauen und der ,Sinn` eines Gesprächs konstituiert sich, anders als in unidirektionalen Kommunikationspraxen, schlussendlich interaktiv als kommunikatives Konstrukt (vgl. Müller 2001; Tiittula 
2001; Brinker/Sager 2006, 127-132). Für unseren Zusammenhang heißt das konkret, dass Wissen durch Sprache im Gespräch kollaborativ konstituiert wird. Teilt man also die Hypothese, dass das ,Web 2.0 Dialog sei“, muss man diese konstitutiven Merkmale des Dialogs mit in die Überlegungen einbeziehen, da diese damit zugleich konstitutiv sind für die Kommunikation in und mit Sozialen Medien. Das hat unmittelbare Konsequenzen für die Unternehmenskommunikation, die immer zielorientiert daraufhin ausgerichtet ist, ein spezifisches Fremdbild (Image) eines Unternehmens, einer Marke usw. evozieren bzw. stabilisieren und mit einem spezifischen Selbstbild (Identität) (zu dieser spezifischen Terminologie in der Unternehmenskommunikation vgl. Kap. 2.2) in Einklang bringen zu wollen. Anders als unter Bedingungen unidirektionaler Massenkommunikation steht sie im Web 2.0 vor der Herausforderung, dieses Ziel gemeinsam im Dialog mit einem Gegenüber erreichen zu müssen.

Unter so genannten Sozialen Netzwerken werden verschiedene Dienste gefasst, von denen die größten strukturell als Microblogging-Dienste verstanden werden können. $\mathrm{Zu}$ den bekanntesten dieser Netzwerke gehören heute Facebook und mit geringerer Reichweite bzw. spezieller Ausrichtung Google Plus. Kennzeichen dieser Dienste ist, dass sie zum einen seit mehreren Jahren ihre Angebote inhaltlich massiv ausbauen und alle Formen der Social Media in das eigene Angebot $\mathrm{zu}$ integrieren suchen. So kommen Sharing-Plattformen für Videos (YouTube mit einer starken Einbindung in Google Plus), Bilder (Instagram zu Facebook bzw. Picasa integriert in Google Plus) sowie aktuell Chattingdienste (WhatsApp für Facebook und Google Hangouts für Google Plus) dazu. Zum anderen baut man seine Präsenz für unterschiedliche technische Plattformen aus - längst sind nicht mehr stationäre oder transportable Rechner das Ziel, sondern mobile Systeme auf Tablet-, Phablet- oder Smartphone-Basis, für die man auch eigene Betriebssysteme erfolgreich etabliert hat (Android für Google) oder dies zumindest versucht (Facebook Home für Facebook). Neben diesen neuen Netzen, die im Web 2.0 durch die Zusammenführung von Angeboten der Social Media entstehen und wachsen, sind andere Angebote noch weitestgehend unabhängig wie etwa der Microblogging-Dienst Twitter oder die Sharing-Plattform Pinterest oder die Blog-Plattform Wordpress, um einige Beispiele zu nennen. Im Kontext dieses Artikels, in dem so genannte Corporate-Blogs stärker in den Vordergrund rücken, sind diese Netzwerke und ihre Angebote insofern relevant, als (Corporate) Blogs nicht allein in der so genannten ,Blogosphäre‘ (vgl. Androutsopoulos u. a. 2006; Heidegger 2004; Ainetter 2006; Hess-Lüttich 2007; Porombka 2012 und Zintzen 2012) oder im Verbund mit anderen Online-Angeboten eines Unternehmens stehen (vgl. Kreutzer 2012, 102), sondern sich der Angebote Sozialer Netzwerke bedienen, um kommunikativ im Web 2.0 anschlussfähig zu sein. 


\section{Markenkommunikation im Web 2.0}

\subsection{Dialogizität}

Als wesentliches Kennzeichen von Social Media wurde die Dialogizität benannt. In diesem Kapitel werden die linguistischen Grundlagen eingehend vorgestellt, die herangezogen werden können, um Dialogizität als grundlegendes Prinzip von Social Media und damit als Herausforderung für die Unternehmenskommunikation zu beschreiben, wenn „Online-Medien in den kommunikativen Auftritt eines Unternehmens“ eingebunden sind (Kreutzer 2012, 102). Häufig ist in Darstellungen zur Unternehmenskommunikation die Rede davon, dass spezifische ,Instrumente eingesetzt“ werden, um spezifische ,Ziele‘ zu erreichen. Konzeptuell liegen diesem Sprachgebrauch Modelle zu Grunde, die die Unternehmenskommunikation als monologisch und unidirektional begreifen. Auch wenn es nach Lakoff (u. a. Lokoff/Wehling 2009) ein Fehler ist, diese hier überhaupt zu nennen, so sei es exemplarisch doch getan: Das „mathematische Modell“ nach Shannon/Weavers (vgl. zusammenfassend z. B. Krallmann/Ziemann 2001) oder das „Vier-Seiten-/Ohren-Modell“ bzw. „Kommunikationsquadrat“ Friedeman Schulz von Thuns (frühe Fassung Schulz von Thun 1981, aktuell Schulz von Thun [o. Datum]) konzeptualisieren den Kommunikationspartner als Empfänger einer Botschaft. Salzer $(2011,9)$ trat vehement für einen „theoretischen Pluralismus im Feld der Unternehmenskommunikation“ in ebendieser Frage ein und Kreutzer (2012) sucht dies perspektivenreich in Bezug auf sein Thema, die so genannte Online-Kommunikation, einzulösen. Doch selbst bei Kreutzer zeigt sich, dass die Lösung von einem Paradigma des Sender-Empfänger-Modells schwer fällt, obwohl eine differenziertere Beschreibung der verwendeten Übertragungstechniken, d. h. der „Instrumente der Unternehmenskommunikation“ vorliegt (vgl. etwa Dialogkommunikation und Netzwerkkommunikation bei Bruhn 2010, 26 f.), wie kurz gezeigt werden soll.

(1) In Bezug auf Online-Kommunikation unterscheidet Kreutzer (2012, 102; Hervorhebung A. L.) etwa zwischen Push- und Pull-Kommunikation: Pushkommunikation spreche Kunden an, ,die Informationen, aus dem Internet herausziehen“ “ müssen; für Pullkommunikation sei ein ,aktiv lesender Nutzer gefordert“. (2) Weiter postuliert Kreutzer, dass Social Media „nicht als weiterer reiner Verkaufs-, Werbe- oder PR-Kanal missverstanden werden“ (Kreutzer 2012, 331; Hervorhebung A. L.) dürfe und dass folgende „Grundprinzipien der Kommunikation in den sozialen Medien“ zu befolgen seien: „Ehrlichkeit/Authentizität, Offenheit/Transparenz, Kommunikation auf Augenhöhe, Relevanz und Kontinuität/Nachhaltigkeit“ (Kreutzer 2012, 335). In Bezug auf die Art und Weise der Kollaboration in den sozialen Netzwerken und Medien nach dem KISS-Prinzip (keep it small/short and simple) (3) unterscheidet Kreutzer (2012, $332 \mathrm{f}$.$) z. B. zwischen kollaborativ und interaktiv wie folgt: Kollaboration bezeichne$ die Zusammenarbeit von Nutzern im oder gegen das Interesse eines Unternehmens 
oder einer Marke, Interaktion bezeichnet den Austausch von Nutzern untereinander und/oder mit dem Unternehmen.

Diese Auswahl an Differenzierungen einer progressiven Darstellung wie der Kreutzers soll exemplarisch zeigen, dass selbst hier noch ein Modell von Kommunikation zu greifen ist, in dem das Unternehmen als Sender und der Mitarbeiter/Kunde/ Shareholder usw. des Unternehmens letztlich als Empfänger konzeptualisiert ist, auch wenn Kreutzer (2012, 332) etwa vom „Prosumenten“ (Kofferwort aus Produzent und Konsument) spricht. Auch die Adaption der Grice'schen Konversationsmaximen kann hier nicht als Gegenargument gelten, da diese sprechakttheoretisch orientiert sind (zur vgl. Einführung Hindelang 2010), d. h. im schlimmsten Falle Sprache verkürzt als Instrument und das Gegenüber einer Sprechhandlung nur als Rezipient betrachten. Anders wird dies in der Gesprächsanalyse gehandhabt (vgl. Rolf/Hagemann 2001; Schwitalla 2001), in der man die Konversationsmaximen um Gesprächsmaximen ergänzt, die konstitutive Merkmale des Gesprächs berücksichtigen (vgl. Kindt 2001):

- In der Regel spricht nur ein Dialogteilnehmer,

- jeder Teilnehmer am Dialog hat das Recht, den nächsten Gesprächsschritt zu beanspruchen und

- dieser Anspruch darf nur dann durchgesetzt werden, wenn der nächste Gesprächsschritt nicht reserviert ist (vgl. Rath 2001; Gruber 2001).

Das Missverständnis in konzeptuell unidirektional ausgerichteten Modellen und damit auch unternehmerischen Kommunikationsstrategien ist, dass man in Social Media das Gegenüber nicht nur auf,Augenhöhe“ mit einer ,Sprechhandlung ' zur Erreichung eines kommunikativen Ziels konfrontiert, sondern ,kollaboriert', ob gewollt oder ungewollt. Social Media macht das Gegenüber zum Sprecher mit eigenen Zielen. Dass eines der basalen Prinzipien von Social Media Dialogizität sei, heißt dabei aber nicht nur, dass man sich irgendwie ,austausche' und ,interagiere', sondern dass man gemeinsam arbeitet und aushandelt, was Ergebnis eines Gesprächs sein soll (vgl. Müller 2001). Das Wissen, das durch Sprache konstituiert wird, wird hier gemeinsam erarbeitet, da die Gesprächsmaximen Ansprüche auf Teilhabe am Gespräch gewähren. Das schließt auch alle unternehmerisch relevanten Themen ein, die zum Inhalt solcher Gespräche werden, die im Web 2.0, wie Kreutzer $(2012,335)$ bemerkt, auf „Augenhöhe“ stattzufinden haben.

Für die Beschreibung der Dialogizität von Social Media ist allerdings nicht nur die Frage des Verhältnisses der Kommunikationspartner von Bedeutung; aus linguistischer Perspektive werden unterschiedliche Parameter für eine Analyse relevant. Wie weit diese ausgreifen, kann man sich an den „Fragen der Soziolinguistik“ leicht vergegenwärtigen: Wer spricht was und wie mit wem in welcher Sprache und unter welchen sozialen Umständen mit welchen Absichten und Konsequenzen? (vgl. Fishman 1972, 15). Fishmans Fragen rufen zum einen gesprächsinterne Merkmale, die sich auf Inhalt, die Struktur des Gesprächs und die Gesprächssteuerung beziehen (das Was 
und das Wie des Gesprächs), und gesprächsexterne Merkmale, die die kommunikativen Bedingungen und die situative Einbettung des Gesprächs betreffen (wer spricht mit wem unter welchen sozialen Umständen), auf. Das Wie des Gesprächs und die Frage nach den Absichten eines Sprechers zielen, von strukturellen Differenzierungen auf sprachlicher Ebene abgesehen, zum anderen auf die Sprechereinstellungen, Erwartungserwartungen (vgl. einführend Hohm 2006, 136f.) des Sprechers vom Gegenüber, Sprecherrollen und damit auch auf die so genannten Konversationsmaximen von Grice (die auch Kreutzer 2012, 335 in seinen „Grundprinzipien der Kommunikation in sozialen Medien“ wiederholt). Die Frage nach den Konsequenzen des Gesagten und damit des Gesprächs setzt eine Maxime voraus, die für die hier thematisierte Dialogizität von Social Media höchste Relevanz hat. Sie ist den Adaptionen der vier Konversationsmaximen in der Unternehmenskommunikation beizustellen. So einfach sie klingt, ist sie dennoch kommunikationsstrategisch höchst brisant und hat in der Forschung zur Unternehmenskommunikation bisher eine nachgeordnete Rolle gespielt: „Rechne mit einer Antwort“.

Neben diesem sehr groben Raster, das der Kategorisierung von Gesprächen dienen kann, hat sich recht früh in der Gesprächsanalyse ein Set an Variablen etabliert, anhand derer sich Gespräche und Dialoge differenzieren lassen (vgl. nach Brinker/Sager 2006, 115-119):

Gesprächsextern: Kommunikationsort, Teilnehmerzahl, Intentionen der Kommunikationspartner, Relation Thema - Sprecher, Öffentlichkeitsgrad, Situationsvertrautheit, Zeitpunkt und Dauer der Kommunikation, Inszeniertheit/Spontaneität der Kommunikation.

Gesprächsintern: Grundaufbau (Einleitung, Begrüßung; Hauptteil; Schlussteil, Verabschiedung), Thematik, Themafixierung, Themenentfaltung (deskriptiv, explikativ, argumentativ; narrativ), Verhältnis von Thema und äußerer Sprechzeit-Welt, Gesprächssteuerung (vgl. unten Abb. 1).

Medialität: Technisches Übertragungsmedium, Medialität der Sprache (mündlich/schriftlich).

Anzumerken ist freilich, dass sich unterschiedliche Variablen unmittelbar auch auf andere Merkmale auswirken: Verfolgen bspw. zwei Kommunikationspartner das Ziel, sich gegenseitig von einem Erlebnis zu erzählen, ist die Themenentfaltung meist narrativ. Eine dieser Variablen, die in Anlehnung an Brinker/Sager 2006 gegeben wurde, ist die der möglichen Dialogsteuerungen, auf die auch in der exemplarischen Analyse einzugehen sein wird: 


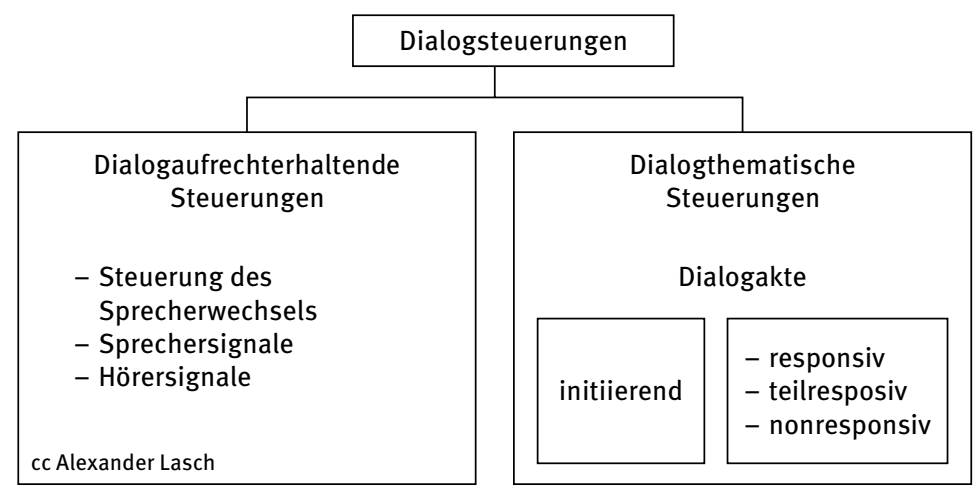

Abb. 1: Begriffsschema zur Dialogsteuerung auf der Basis von Schwitalla 1976, vgl. weiter Müller 2001 und Tiittula 2001.

Betrachten wir Social Media als wesentlich für das Web 2.0 und begreifen wir Dialogizität als Prinzip des Web 2.0 und damit Dialogizität als eines der konstitutiven Merkmale von Social Media, dann ist mit komplexeren Modellen wie den vorgestellten verbale Interaktion, also Kommunikation, $\mathrm{zu}$ analysieren und unter Beachtung genannter Faktoren strategisch zu planen.

\subsection{Selbstbild und Fremdbild (Corporate Identity und Corporate Image)}

Die Begriffsverwendung von Image weicht in der Unternehmenskommunikation stark vom Imagebegriff ab, der in der Linguistik über die Soziologie etabliert ist (vgl. etwa Goffmans Arbeiten zur Imagearbeit, exempl. 1996, 10-53). Dieser Artikel wird sich allerdings zunächst am Gebrauch des Imagebegriffes in der Unternehmenskommunikation orientieren. Ein Diktum der Forschung lautet, dass es eine der wesentlichen Aufgaben von Unternehmenskommunikation sei, die Bedingungen der Kommunikation, der Selbstdarstellung, eines bestimmten Selbstbildes (Corporate Identity) $\mathrm{zu}$ analysieren (vgl. verschiedene Ausdifferenzierungen bei Gioia u. a. 2000, Birkigt/ Stadler/Funk 2002, Schmid/Lyczek 2008), zu beschreiben und aus den Ergebnissen eine Strategie dieser Kommunikation des Selbstbildes abzuleiten, um ein bestimmtes Fremdbild (Corporate Image) zu evozieren (vgl. Schmid/Lyczek 2008, Mast 2010, 46; der Begriff der Reputation wird hier nicht aufgenommen oder diskutiert; vgl. Burel; Mast beide in diesem Band).

Das Identitätskonzept als Selbstbild wird in diesem Beitrag weder in Bezug auf die Genese noch im Hinblick auf Soll- bzw. Ziel-Zustand differenziert und kritisch reflektiert (vgl. Bungarten 2005 und Seidler 1997, Burel in diesem Band). Dies ist der Knappheit der Darstellung und dem ausgewählten Gegenstand geschuldet. In der 
externen Unternehmenskommunikation wird im besten Falle ein (!) angestrebtes Selbstbild kommuniziert, ob dieses von einer (idealisierten) Person (als Unternehmen) oder durch ein Kollektiv getragen wird, ob es Soll- oder Zielzustand darstellt oder durch welche Prozesse es entsteht, ist dementsprechend aus dieser Perspektive von nachgeordnetem Interesse. Weiter ist die Scheidung von Identität (Selbstbild) und Image (Fremdbild) eine analytische, da Elemente des ausgehandelten Images durchaus Eingang in das Selbstbild finden und umgekehrt (vgl. Kastens 2008) - sie bilden die beiden Seiten einer Medaille von dem, was die Soziologie einheitlich Image nennt (vgl. zur Adaption in der Linguistik bspw. Holly 2001 genau in diesem Sinne).

Das Selbstbild (CI) wird in der relevanten Literatur noch weiter differenziert in Corporate Design (CD), Corporate Communications (CCom) und Corporate Behavior (CB) (vgl. Salzer 2011, 11 oder allgemeiner Bruhn 2010, 89), darüber hinaus nicht systematisch erweitert um Corporate Culture (CCult; Mast 2010 und in diesem Band mit dem Fokus auf kommunizierte Wertvorstellungen) sowie Corporate Wording (CW; Förster 1994 und in diesem Band). Die Forschung zur Unternehmenskommunikation nahm vor allem Aspekte der CCom in den Blick (vgl. Mast zu CCult und Förster zu CW in diesem Band), während CD und CB eher im Hintergrund zu stehen scheinen, wenn die Etablierung einer CI beschrieben werden soll. Die Vernachlässigung der Kommunikationsumgebung (CD) und des Kommunikationsverhaltens (CB) in der Forschung und in der strategischen Ausrichtung der Unternehmenskommunikation fällt besonders im Hinblick auf die Social Media ins Gewicht, die Kommunikationsumgebungen und -gelegenheiten an spezifischen Orten und unter spezifischen Gesetzmäßigkeiten offeriert (vgl. Kap. 2.3). Zum anderen ist die Planung von unidirektional organisierten Sinnstiftungsprozessen eines Selbstbildes (CI) in ,Gesprächsumgebungen` wie Social Media ergebnisorientiert nur bedingt möglich, da sich diese im ,Gespräch`zu unerwartet entwickeln können, um erfolgreich zu prognostizieren, welche Schritte nötig sind, Selbstbild und Fremdbild in Einklang zu bringen (vgl. zu diesen Aspekten ausführlich Burel in diesem Band). Unternehmen laufen tendenziell Gefahr, mit ihrer Imagearbeit ungewollt ein negatives Image zu erzeugen, wenn sie das Selbstbild eines Unternehmens oder die Identität eines Produktes zum Thema eines ,Gesprächs“ auf den Kanälen des Web 2.0 machen.

Das hängt mit der Dialogizität von Social Media unmittelbar zusammen: Auf eine zurückweisende Antwort, die sich auf den Versuch der Etablierung eines bestimmten Selbstbildes bezieht, wird dann in den meisten Fällen nicht adäquat reagiert werden, wenn der Kommunikationsstrategie eines Unternehmens ein unidirektionales Kommunikationsmodell wie das der Sprechakttheorie oder das einfache SenderEmpfänger-Modell zu Grunde liegt, wobei es unerheblich ist, wie man das Selbstbild eines Unternehmens konzeptualisiert (vgl. zusammenfassend Burel in diesem Band, Kap. 1.5). Wird das Selbstbild (Identität) zum Thema in den Kanälen von Social Media, dann wird nicht nur diese Inszenierung des Selbstbilds zur Etablierung eines Fremdbildes (Images) beitragen, sondern auch das Gespräch darüber, zu dem alle Gesprächspartner gemeinsam beitragen: Es wird ein Wissen über ein Unternehmen in 
einem Aushandlungsprozess von mehreren Teilnehmern sprachlich konstruiert und prozessiert (vgl. dazu Hundt 2009 und Ebert/Konerding 2008).

Dieses Prinzip steht allerdings quer zum unternehmerischen Ziel und zur Vorstellung von Unternehmenskommunikation, mittels „Instrumenten der Unternehmenskommunikation“ (Bruhns) ein einheitliches Fremdbild (Image) eines Produkts, einer Marke oder eines Unternehmens zu etablieren, das möglichst viele Merkmale des inszenierten bzw. ausgehandelten Selbstbildes aufweist.

\subsection{Das Corporate Blog als virtueller Kommunikationsraum (Corporate Design)}

Social Media unterliegt nicht nur dem Prinzip der Dialogizität und stellt damit Kommunikation unter spezifische Bedingungen, sondern Social Media etabliert auch Räume, die diesen Bedingungen und weiteren Gesetzmäßigkeiten Rechnung tragen. Mit diesem Raum ist ein virtueller Ort gemeint, an dem Kommunikation zwischen verschiedenen Teilnehmern als Gespräch stattfindet. Über den Ort und seine Charakteristika kann der Zugang zum Gespräch, die Anzahl der Teilnehmer, die Hierarchisierung und Rollenzuweisung von Gesprächspartnern, Privilegien in Bezug auf die Gesprächssteuerung und damit Hierarchien zwischen Gesprächspartnern sowie die Themenwahl geregelt werden. Der Ort, an dem Kommunikation stattfinden kann, wird zum einen durch die medialen Gegebenheiten und zum anderen durch das Corporate Design (CD) eines Unternehmens vorstrukturiert.

Auch wenn es reizvoll wäre, verschiedene Formen von Social Media vor diesem Hintergrund $\mathrm{zu}$ analysieren (die Linguistik entdeckt das Themenfeld nur langsam, vgl. aber Androutsopoulos 2007, 2014; Moraldo 2009; Porombka 2012, Dürscheid/ Frick 2014; Dürscheid/Brommer 2013; Barnes 2013; Eisenlauer 2013; Schlobinski/ Siever 2000; Runkehl/Schlobinski/Siever 2013; Burghardt u. a. 2013; Frick 2014) und typologisch im Hinblick auf die Erfordernisse der Unternehmenskommunikation hin zu beschreiben, konzentriert sich dieser Beitrag auf Corporate Blogs und Firmenweblogs als digitale Publikations- und Kommunikationsplattformen. Die Forschungslage $\mathrm{zu}$ diesen Corporate Blogs ist noch recht überschaubar; erste Ergebnisse wurden vor allem aus den Bereichen der Kommunikationswissenschaft (Zerfaß 2005; Mast 2010) und Wirtschaftswissenschaft (Bruhns 2010; 2011; Kreutzer 2012) vorgelegt, die Sprachwissenschaft wendet sich eher grundsätzlich dem Thema der ,computervermittelten Kommunikation“ (CMC) zu (vgl. exempl. Androutsopolous 2005; 2007; Androutsopoulos/Kasten 2005; Androutsopoulos u. a. 2006; Dürscheid 2006, 2009; Dürscheid/Ziegler 2002, Dürscheid/Brommer 2009, 2013; Puschmann 2010; Anders/ Hundt/Lasch 2011; Herring/Stein/Virtanen 2013; Marx/Weidacher 2014; Siever 2013a und b; 2014a und b). Zur Kommunikation via Blog liegen bisher nur wenige Arbeiten vor (z. B. Fraas/Barczok 2006; Handler 2006), die meisten mit literaturwissenschaft- 
licher Orientierung im Hinblick auf Inszenierung von Autorschaft (u. a. Porombka 2012; Zintzen 2012; Ainetter 2006; Heidegger 2004 oder Schuster 2013).

Corporate Blogs gehören zur „(Online-)Visitenkarte eines Unternehmens“ (Kreutzer 2012, 107) und sind den „Kommunikationsmedien“ (sic!, neben „Kooperationsmedien“ und „Content-Sharing-Medien“) von Social Media zuzurechnen (vgl. Kreutzer 2012, 331 f.); Übersichten über verschiedene Blogformate vom Knowlegde-Blog und Collaboration-Blog (interne Kommunikation) über Service-Blogs, Campaigning-, Issues-, Product/Brand-, Customer-Relation- bis hin zu Crisis-Blogs (externe Kommunikation) (Bruhn 2010, 479) mit den aus der Sprechakttheorie übernommenen Funktionen der Information, der Persuasion und der Expression liegen vor. Die Einteilung ist im Übrigen wieder ein Hinweis auf die ,instrumentelle Orientierung‘ der Unternehmenskommunikation.

Corporate Blogs dienen Unternehmen dazu, um „mit unterschiedlichen Stakeholdern in einen Dialog zu treten“ und „dabei zunächst selbst fest[zu]legen, welche Neuigkeiten distribuiert und welche aus Unternehmenssicht wichtigen Themen angestoßen werden sollen.“ (Kreutzer 2012, 346). Weiter „öffnet sich das Unternehmen auch hier den Fragen der Leser, die kompetent und ehrlich [zu ergänzen ist: und auf Augenhöhe] zu beantworten sind.“ (Kreutzer 2012, 349). Allen Bekenntnissen zum Trotz wird jedoch weiter an der Idee festgehalten, Gespräche und Dialoge, Kommunikation im Web 2.0, sprechakttheoretisch modellieren zu können. Auf Corporate Blogs bspw. können, so Kreutzer 2012, 350 (vgl. auch Bruhn mit der Übersicht über die ,Sphäre der Corporate Blogs'):

Interessierte ,live' miterleben, womit sich das Unternehmen beschäftigt. Diese Offenheit des Unternehmens, seine Kunden und Stakeholder über laufende Aktionen zu informieren, kann idealerweise die Bindung an das Unternehmen fördern und durch eine aktive Teilnahme, zumindest in Grenzen, zu seinen Gunsten beeinflussen. (Hervorhebung A. L.).

Der Unternehmenskommunikation liegt die Vorstellung zu Grunde, dass man mit Corporate Blogs ,ein Instrument‘ der Unternehmenskommunikation in der Hand habe, um ,Shareholder` zu ,informieren` und mit ihnen in ,Dialog‘ treten zu können, ohne dabei auf die Mittlerrolle traditioneller (Massen-)Medien angewiesen zu sein. Gesprächspartner auf ,Augenhöhe‘ werden als ,interessierte Miterlebende‘ konzeptualisiert, die durch ausgestellte ,Offenheit‘ so ,beeinflusst‘ werden können, dass sie eine ,Bindung‘ zum Unternehmen aufbauen. Dass sich jedoch die Hoffnung erfüllt, dass man durch ,Information“ ,Shareholder‘ besser ,binden“ könne, ist ausgehend von den theoretischen Prämissen der Sprechakttheorie und der Interaktionsanalyse schlicht unwahrscheinlich. Aus linguistischer Sicht gelingt dies nicht durch ,informierende‘, sondern durch ,expressive‘ Sprechakte, von dialogischen Sinnstiftungsprozessen noch nicht einmal gesprochen (vgl. Müller 2001). Weiter verkennt man mit einer solchen Einschätzung die Kommunikationsordnungen eines Blogs, derer 
man sich besser bewusst sein sollte, wenn man Kommunikationsstrategien für Social Media-Formate entwickelt.

\subsection{Kommunikationsstrategien (Corporate Behavior)}

Die folgenden Beschreibungen bauen darauf auf, dass die Basis für textlinguistisch und gesprächslinguistisch motivierte Studien in der Sprechakttheorie begründet liegt und daher beide pragmatisch ausgerichtete Ansätze meist auf ein gemeinsames Inventar und gemeinsame Kategorisierungen zurückgreifen, um Texte bzw. Gespräche zu analysieren (vgl. Brinker u.a. 2000, Brinker u.a. 2001; Birkner/Janich im Druck; einschlägig zur Gesprächslinguistik Brinker/Sager 2006 und zur Textlinguistik Brinker 2010).

Die Charakteristik eines Blogs für die externe Unternehmenskommunikation ist, dass Kommunikation an einem bekannten Ort mit einer offenen und teils anonymen Zahl an mehr oder weniger professionellen Teilnehmern öffentlich stattfindet - dabei kann nicht vorausgesetzt werden, dass alle Teilnehmer mit den Regeln der Kommunikation im Netz und spezieller auf Blogs hinreichend vertraut sind. Den Zugang zum Blog kann der Betreiber steuern; hierfür stehen verschiedene Regelmechanismen zur Wahl, die alle Formen und Arten der Partizipation am Gespräch betreffen: Mittels der Festlegung, ob Nutzer unter Klarnamen statt Pseudonymen am Gespräch teilnehmen dürfen (unter der belastbaren Annahme, dass scheinbare Anonymität bisweilen ausgenutzt wird), der Angabe von E-Mail-Adressen, diversen Optionen für die Freischaltung der Kommentare von Nutzern, der Sperrung von Nutzern, der Einladung zur Moderation von Kommentaren, der Vergabe des Rechts auf die Mitarbeit am Blog (Korrektur, Verfassen von Blogartikeln) bis hin zu umfassenden Administratorrechten steuert und selegiert der Betreiber den Zugang zum virtuellen Kommunikationsraum. Zeitpunkt und Dauer von Mehrpersonengesprächen auf Blogs können nicht vorhergesehen werden, allein der initiierende Gesprächsschritt ist im Idealfall planbar, faktisch bestimmt der Betreiber des Blogs aber auch, wann ein Gespräch endet. Anders als im Gespräch auf einem Blog suggeriert werden soll, sind die Machtverhältnisse klar geregelt: Der Betreiber des Blogs setzt die Regeln und achtet zugleich auf ihre Einhaltung. Er öffnet und schließt den Kommunikationsraum Blog nach Belieben, initiiert Gespräche und gibt die Themen für die Gespräche vor, reguliert die Teilnahme am Gespräch und das Kommunikationsverhalten im Gespräch durch Moderation, setzt Beginn und Ende des Gesprächs. Die strukturellen Voraussetzungen zeigen, dass hier nicht auf ,Augenhöhe‘ kommuniziert wird, sondern dass wie auf jedem Blog eine starke Asymmetrie zwischen den Gesprächsteilnehmern vorliegt. Die initiierenden Gesprächsschritte sind inszeniert, da sie wenigstens einer bestimmten Intention und auch Funktion (Kommunikationsstrategie) folgen und der CCom bzw. dem CB oder dem CW unterworfen sind; für das disperse Gegenüber können diesbezüglich keine differenzierenden Aussagen gemacht werden. Abgesehen davon kann in Bezug auf 
die Vorbereitetheit und die Spontaneität der Gesprächspartner grundsätzlich gelten: Je konfliktträchtiger das Thema, desto vorbereiteter gehen alle Teilnehmer in ein thematisches Gespräch.

Aus diesen, gesprächsexternen Faktoren' lassen sich Rückschlüsse auf ,gesprächsinterne Merkmale، der Blog-Kommunikation ziehen. Der Grundaufbau bildet die Machtverhältnisse ab: Der Betreiber initiiert ein Gesprächsthema mit einem Blogartikel, er lädt zum Gespräch ein, wenn die Kommentar- und Sharing-Optionen für einen Artikel freigegeben werden. Dieser Artikel ist als initiierender Dialogakt als Teil der dialogthematischen Steuerung aufzufassen. Das eigentliche Gespräch entwickelt sich auf der Basis dieser Themensetzung, der Themenfixierung. Von der Art der Thematik und der Art des initiierenden Gesprächsschrittes durch den Betreiber, der damit eine bestimmte Haltung und Perspektive zum gewählten Thema einnimmt, sind unterschiedliche Themenentfaltungen im sich in den Kommentaren auf dem Blog oder an anderen Kommunikationsorten der Social Media wie Sozialen Netzwerken entwickelnden Gespräch abhängig: Auf die Initiierung eines Dialogs folgen (voll-)responsive (Thema, Gesprächshaltung und Positionierung des Gegenübers zum Thema werden angenommen), teilresponsive (Thema und/oder Gesprächshaltung und/oder Positionierung des Gegenübers zum Thema werden angenommen) oder nonresponsive Dialogakte (weder Thema noch Gesprächshaltung noch Positionierung des Gegenübers zum Thema werden angenommen) (vgl. Schwitalla 1976). In der Hinführung war davon gesprochen worden, dass es ratsam sei, den Imperativ „Rechne mit einer Antwort!“ in kommunikationsstrategischen Planungen zu berücksichtigen. Genau diese Stelle, an der der Sprecherwechsel zu den Besuchern des Blogs gewünscht ist, ist der sensible Punkt in einem Gespräch. Hier kann der Betreiber vor allem in kritischen Gesprächssituationen zeigen, wie und ob er seine strukturell vorgegebene Machtposition einsetzt und ausnutzt, oder ob der Inszenierung von ,Kommunikation auf Augenhöhe‘ der Vorzug gegeben wird.

Das Corporate Blog ist ein stark regulierter virtueller Kommunikationsraum, in dem Gespräche stattfinden können, die durch den Betreiber des Blogs initiiert werden (vgl. Fraas/Barczok 2006; Handler 2006; Porombka 2012): Das Blog folgt dem Prinzip der Dialogizität von Social Media. Vor dem Hintergrund der genaueren Beschreibung der Struktur des virtuellen Kommunikationsraums Blog ist eine Kommunikationsstrategie zu entwickeln. Diese muss aufzeigen, wie und ob es ratsam sei, die Inszenierung des Selbstbilds zur Etablierung eines Fremdbildes (Images) in Aushandlungsprozessen von Gesprächen zu platzieren.

Hier wird die Ansicht vertreten, dass wesentliche Aspekte der Corporate Identity oder des Bildes des Unternehmens von einem Produkt, einer Marke usw. in einem virtuellen Kommunikationsraum wie einem Corporate Blog zwar sichtbar sein sollten (Corporate Design), aber im Interesse des Unternehmens, wie gezeigt, nicht explizit Gegenstand lediglich ,informierender' initiierender Dialogakte werden sollten (Corporate Behavior), um Aushandlungsprozesse in Gesprächen in Bezug auf das Fremdbild (Image), Prozesse der Wissenskonstitution und -stabilisierung, nicht noch 
direkt anzustoßen. Berühren sprachlich ausgehandelte und konstruierte Fremdbilder (Images eines Unternehmens, einer Marke, eines Produktes) das Selbstbild, dann hat der Betreiber eines Blogs zahlreiche Möglichkeiten, um auf diese Aushandlungsprozesse zu reagieren. Exemplarisch sind hier sieben aufgeführt, wobei (1) und (2) das Fremdbild (Image) positiv beeinflussen, (3) positive wie negative Folgen haben kann und (4), (5), (6) sowie (7) das Fremdbild (Image) mit Sicherheit negativ beeinflussen, da sie das Prinzip von Dialogizität von Social Media grundsätzlich in Frage stellen - alle Optionen sollten in einer Richtlinie zum Coporate Behavior (CB) bewusst gemacht werden. Der Betreiber kann

(1) einen oder mehrere Gesprächsschritte von kommentierenden Nutzern (auch von anderen Social Media-Präsenzen) in einen neuen Blogartikel (bottom-up) aufnehmen. Dieser Artikel inszeniert die Gesprächsinitiierung als ,Fortsetzung“, was unter den Kommunikationsbedingungen des Blogs im Speziellen und Social Media im Allgemeinen positiv bewertet wird. Der Betreiber schiebt damit die Verantwortung für die Behandlung des Themas und die Gesprächseröffnung von sich auf das Gegenüber und zeigt, dass er seine Machtposition bei der Gesprächsinitiierung nicht ausnutzt. Er zeigt sich als kollegial, auf Augenhöhe stehend.

(2) Feedback zu Themen und Fragen einholen (Umfragen, Gewinnspiele usw.), die in Gesprächen auf dem Blog ausgehandelt werden und das Selbstbild des Unternehmens betreffen. Die Darstellung von Ergebnissen von Umfragen lässt sich analog $\mathrm{zu}$ (1) als Gesprächsfortsetzung inszenieren.

(3) sich in die Aushandlung einmischen und seine Position noch einmal explizieren, dezidiert zu positiv wie negativ evaluierenden Kommentaren Stellung beziehen. Das wird von Nutzern in aller Regel zwar positiv bewertet, ist aber in Bezug auf den Versuch der Korrektur des Fremdbildes nur selten erfolgreich; die strukturell erfolgversprechendere Antwort ist längerfristig ein weiterer Artikel. $\mathrm{Zu}$ bedenken ist weiter, dass es in den Diskussionen auch zu Konflikten kommen kann, da der Betreiber meist verschiedene Rollen (Autor, Moderator, Administrator) innehaben kann. Dezidiert sei an dieser Stelle gesagt, dass dieser Artikel nicht auf virales Marketing, dessen Enttarnung und dementsprechende negative Konsequenzen eingeht.

(4) Aushandlungsprozesse stoppen, in dem er bspw. Kommentare herausstellt, korrigiert, editiert, löscht oder Nutzer sperrt.

(5) Aushandlungsprozesse vermeiden, indem er keine Kommentare zulässt.

(6) darauf verzichten, weitere gesprächsinitiierende Artikel anzubieten.

(7) das Blog schließen.

Wenn nicht die ,Information‘ über ein Produkt oder eine Marke bzw. die ,Explikation' des Selbstbilds eines Unternehmens aus strukturellen Gründen Thema eines Corporate Blogs sein sollen, was ist dann Inhalt der Gespräche, die die Teilnehmenden ,binden“ sollen? Die Antwort ist linguistisch erwartbar und vor dem Hintergrund der Interaktionsanalyse scheinbar banal: ,Expressive Sprechakte‘ bzw. Gesprächs- 
schritte mit expressiver Funktion haben das Potential, das Gegenüber auf emotionaler Ebene anzusprechen und einzunehmen (CCom), indem sie die Beziehungsebene der Gesprächsteilnehmer in einem Kommunikationsraum, in dem das Selbstbild omnipräsent und damit immer im Blick ist (CD), in den Mittelpunkt stellen (CB) (vgl. Dürscheid/Brommer 2013 zur Adaption des Konzeptes „Freundschaft“ in sozialen Netzwerken). Auf einem Corporate Blog müssen Kommunikationsanlässe geschaffen werden, die Gespräche initiieren, die bestenfalls thematisch ergebnisoffen sind und nur mit geringstem Aufwand gesteuert werden müssen. Dabei muss sich die strategische Ausrichtung der Unternehmenskommunikation an Erwartungserwartungen ausrichten, die der Betreiber vom Gesprächsgegenüber hat, die er selbst mit in die Kommunikation einbringt und auf die er seine gesprächsinitiierenden Akte intentional und funktional ausrichtet.

\section{Exemplarische Analyse}

Für die folgende Analyse können zwei unterschiedliche Szenarien aufgerufen werden, die analytisch gleichermaßen aufschlussreich sind.

Typisch für das eine Szenarium wären konfliktträchtige Gespräche, die sich an krisenhafte Vorfälle anschließen und Teil so genannter und gefürchteter Krisenkommunikation sind. Das sind bspw. Ereignisse, in denen energie- und rohstofffördernde Unternehmen Umweltkatastrophen oder Lebensmittelhersteller Lebensmittelskandale verursachen bzw. sich nicht an allgemein geltende ethische Standards halten. Auf dem Spiel steht immer neben objektivierbaren Verlusten auch der ,des Vertrauens' in das Unternehmen. Gemeint ist damit, dass das ganz wesentlich durch Sprache konstituierte Fremdbild (Image) von einem Unternehmen, einem Produkt, einer Marke durch negative Aspekte, die diskursiv ausgehandelt werden, deutlich abgewertet wird. $\mathrm{Zu}$ befürchten ist, dass Fremdbild und Selbstbild so weit auseinanderdriften, dass der ,Shareholder‘ ein Unternehmen, eine Marke, ein Produkt im buchstäblichen Sinne nicht wiedererkennt, oder dass in das Selbstbild (bei einer kollektiven Identität) negative Aspekte des Fremdbildes aufgenommen und zum Thema unternehmensinterner Auseinandersetzungen werden. Trotz dieser Gefahren sind Unternehmen und Organisationen in krisenhaften Situationen auf den Kanälen von Social Media aktiv, da wie bereits kurz dargelegt, die Reglementierung von Kommunikation und die Ausnutzung der strukturellen Hierarchie zwischen den Gesprächspartnern (CB) auf einem (Corporate) Blog noch ernstere Folgen für das Fremdbild (Image) und damit auch für das Selbstbild (Identität) des Unternehmens mit sich brächte.

Auf der anderen Seite widmete man sich Kommunikationspraxen, in denen erfolgreich die Beziehungsebene thematisch in den Vordergrund gestellt (als Prinzip der CCom) und so ein Gespräch im Angesicht der Marke (als Prinzip des CD) auf einem Corporate Blog geführt wird. Dieses kann das diskursiv ausgehandelte Fremdbild 
(Image) und damit sekundär das Selbstbild (Identität) eines Unternehmens positiv beeinflussen.

Für die Darstellung wählen wir eines der nachfolgenden best-practice-Beispiele aus. Seit Jahren gelten im deutschsprachigen Raum die Corporate Blogs „Das Daimler-Blog“ (http://blog.daimler.de/, Zugriff am 15.07.2014), das „Ritter Sport“-Blog (http://www.ritter-sport.de/blog, Zugriff am 15.07.2014) oder das „Audi Blog“ (http:// blog.audi.de/, Zugriff am 15.07.2014) als sehr gute Beispiele für eine Ergänzung des Online-Portfolios eines Unternehmens durch Corporate Blogs. In den Mittelpunkt der Analyse wird das „Ritter Sport“-Blog gerückt (vgl. Abb.2), das in Kooperation zwischen der „elbkind GmbH“ und der „Pluspol Interactive GbR“ auf der Basis eines älteren Corporate Blogs entwickelt wird.

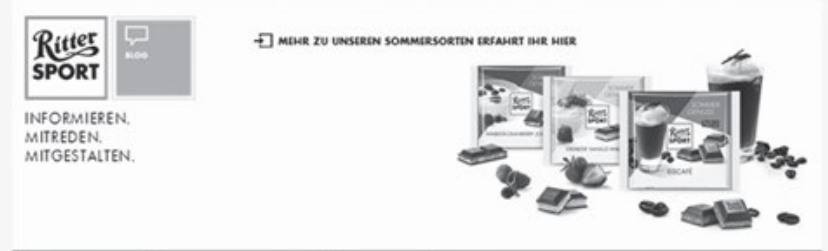

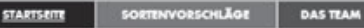

13 DIY: RITTER SPORT Hülle für das Smartphone

und Co. einfach selbstgemacht

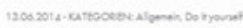

Hey keone sadolond

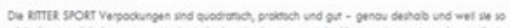

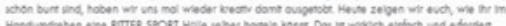

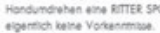

and
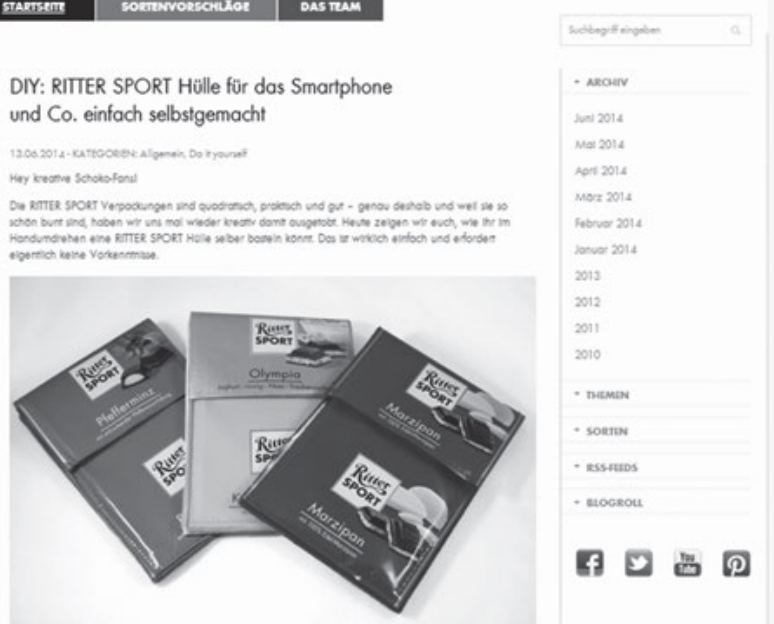

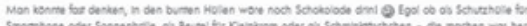

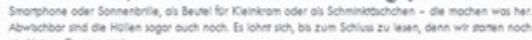

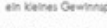

(weteriesen....)

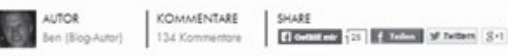

Abb. 2: Startseite des „Ritter Sport“-Blogs

(http://www.ritter-sport.de/blog/, Zugriff am 15.07.2014) 
Laut „elbkind GmbH“ sollten beim Neustart des Corporate Blogs

die Stärken des bisherigen Blogs aufrecht erhalten [sic!], zusätzlich aber auch Einiges [sic!] neu gestaltet werden. Der [sic!] neue Blog ist im Full-Responsive Design gestaltet. (http://elbkind.de/ arbeiten/ritter-sport-3/, Zugriff am 17.12.2014)

\section{Weiter wurde}

[b]esonderer Wert [...] auf den Bereich Sortenvorschläge gelegt - sie sind nun ein zentrales Element auf Blog und Facebook. Mit den Sortenvorschlägen ist ein permanentes CrowdsourcingFeature integriert. In einer Applikation können neue Sorten eingereicht, Name, Layout und Zutaten bestimmt werden - und sofort wird angezeigt, wie die Sorte später aussehen könnte. (http://elbkind.de/arbeiten/ritter-sport-3/, Zugriff am 17.12.2014)

Das Unternehmen steht im inszenierten Dauerdialog mit seinen Kunden in Bezug auf das zentrale Produkt des Unternehmens. Das Blog weist eine typische Struktur auf. Es ist neben der Artikelübersicht im Wesentlichen gegliedert in Kopf (Logo, dreischrittiger Slogan, Seitenhinweise), Seitenregister (Startseite, Sortenvorschläge, Autorenteam) und Suchfunktion, Artikelarchiv, Sharing-Optionen sowie Präsenzen in Sozialen Netzwerken - also andere Formen von Social Media - am rechten Rand des Blogauftritts (Facebook, Twitter, Youtube und Pinterest). Der Kopf ist geprägt durch zwei Logos und einen Leitspruch für das Blog, der rhetorisch als dreischrittige Klimax einzustufen ist. In Bezug auf die sprachliche Detailanalyse wäre anzumerken, dass „Mitlesen“ statt „Informieren“ aus verschiedenen Gründen der Vorzug zu geben wäre (vgl. im Detail zur Untersuchung der sprachlichen Qualität von Firmenauftritten im Internet Anders/Hundt/Lasch 2011). Die Logos des Kopfes und die meisten Designelemente (etwa die Angabe des Datums eines Blogeintrags) weisen auf eine Besonderheit hin, die dem Corporate Design geschuldet ist und Hand in Hand mit dem Corporate Wording (CW) des Unternehmens geht, dessen Slogan für seine wichtigste Marke ist: „Quadratisch. Praktisch. Gut.“

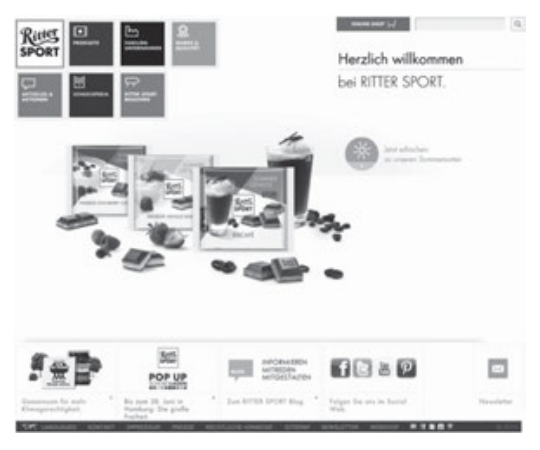

Abb. 3: Deutschsprachige Startseite des Unternehmensauftritts von „Ritter Sport“ (http://www.ritter-sport.de/, Zugriff am 15.07.2014) 
Das zweite Logo „Blog“ scheint auf den ersten Blick dysfunktional zu sein (die Verlinkung führt wie die des Firmenlogos zur Startseite des Blogs), nimmt auf den zweiten Blick aber das Corporate Design des Online-Portfolios des Unternehmens auf (Abb. 3). Die farbige Gestaltung des Corporate Blogs (Abb. 2) ist orientiert am CD für das Produkt des Unternehmens, dem auch das Blog gewidmet ist: „Ritter Sport“-Schokolade.

Im Folgenden wird ein Artikel des „Ritter Sport“-Blogs im Mittelpunkt des Interesses stehen, um zu zeigen, wie der Kommunikationsraum Blog dazu genutzt wird, Gespräche zu initiieren, die hinsichtlich der Gesprächsfunktion als expressiv einzustufen und ergebnisoffen bei minimaler Steuerung sind. Genauer wenden wir uns dem Artikel „DIY [sc. do it yourself]: RITTER SPORT Hülle für das Smartphone und Co. einfach selbstgemacht“ von „Ben“, einem Mitarbeiter der Agentur „elbkind GmbH“, $\mathrm{zu}$ (http://www.ritter-sport.de/blog/2014/06/13/diy-ritter-sport-hulle-fur-das-smartphone-und-co-einfach-selbstgemacht/, Zugriff am 15.07.2014). Informatives Ziel des Artikels ist, zu zeigen, „wie ihr im Handumdrehen eine RITTER SPORT Hülle selber basteln könnt. Das ist wirklich einfach und erfordert eigentlich keine Vorkenntnisse.“ Zunächst erfolgt eine direkte Anrede, die kurze Wiedergabe des Markenslogans und der Hervorhebung des Produktdesigns:

Die RITTER SPORT Verpackungen sind quadratisch, praktisch und gut - genau deshalb und weil sie so schön bunt sind, haben wir uns mal wieder kreativ damit ausgetobt. (http://www.rittersport.de/blog/2014/06/13/diy-ritter-sport-hulle-fur-das-smartphone-und-co-einfach-selbstgemacht/, Zugriff am 17.12.2014)

Danach entspricht der Artikel weitestgehend dem Exemplar der Textsorte „Instruktionstext“ (vgl. Möhn 1991; Rolf 1992; Ehlich u. a. 1994; Schwender 1999; Gülich/ Hausendorf 2000; Heinemann 2000, Motsch 2000; Brinker/Hagemann 2001; Brinker 2010): Es wird das notwendige Material angegeben und dann Schritt für Schritt mit Bebilderung erklärt, wie eine Umverpackung recycelt werden kann. Das Endprodukt ist praktisch und gut, da nützlich, ressourcenschonend und damit umweltbewusst. Weiter entspringt das Endprodukt einer eigenständigen und kreativen Tätigkeit, weiß zu überraschen und ist funktional. Es setzt die Marke und das Markenlogo in Szene, ohne dass über die Marke bis auf die einleitende Wiedergabe des Slogans ein einziges Mal gesprochen worden wäre. 


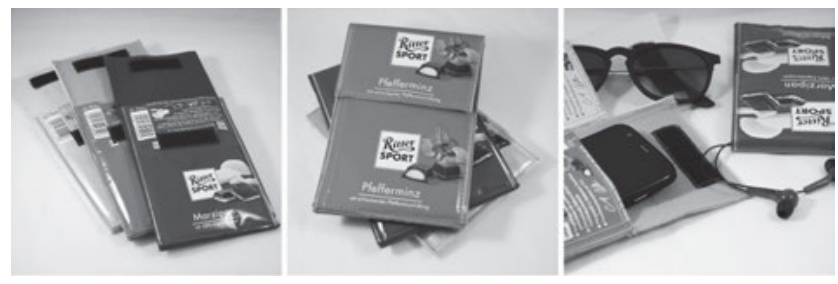

Abb. 4: Das Ergebnis: DIY-Smartphonehülle (http://www.ritter-sport.de/blog/2014/06/13/

diy-ritter-sport-hulle-fur-das-smartphone-und-co-einfach-selbstgemacht/, Zugriff am 15.07.2014)

Der Charakter des „Full-Responsive Designs“ des Blogs ließ sich bisher nur an der Anrede und Einladung zum Mitmachen (gemäß dem Motto des Blogs „Mitgestalten“) ableiten - der Artikel stiftet einen Kommunikationsanlass. Aber der Verfasser tut noch mehr dafür und steuert damit die zu erwartenden Reaktionen. Zum einen wird nach der Instruktion das Gegenüber adressiert (vgl. Hartung 2001) mit einer Frage: „Habt ihr noch weitere Ideen?“ In der Terminologie der Interaktionalen Linguistik wäre dieser Gesprächsschritt als Aufforderung zum Sprecherwechsel im Gespräch zu bezeichnen. Darüber hinaus wird explizit dazu aufgefordert, die eigenen Ergebnisse in den Sozialen Netzwerken zu streuen: „Wir freuen uns riesig, wenn ihr die Hülle bastelt und uns ein Bild eurer genähten Hülle sendet oder auf unsere Facebook-Fanpage postet!“ Im Abschluss des Artikels wird schließlich ein Gewinnspiel bekanntgegeben:

Das Beste kommt zum Schluss: Wir verlosen die drei abgebildeten Hüllen! Da sie handgefertigt sind, ist jede ein Einzelstück, ggf. auch mit kleinen Schönheitsfehlern. Wenn ihr eine Hülle gewinnen möchtet, schreibt uns einen Kommentar: Wieso möchtet ihr die Hülle gewinnen und für was würdet ihr sie benutzen? Bis zum 15.06.2014 um 23:59 Uhr könnt ihr euer Glück versuchen. Unter allen Kommentaren losen wir dann die drei Gewinner aus. Lest bitte auch die Teilnahmebedingungen. Wir drücken euch die Daumen! (http://www.ritter-sport.de/blog/2014/06/13/diy-rittersport-hulle-fur-das-smartphone-und-co-einfach-selbstgemacht/, Zugriff am 15.07.2014)

Wie an dieser kurzen Vorstellung des Artikels deutlich wird, steht die Marke und das Produkt im Hintergrund der Gesprächsinitiierung mittels eines Textes: Es geht um die Umverpackung eines Produktes, die recycelt wird zu einem neuen Produkt, einer Smartphonehülle. Eine ,Information' des ,Shareholder‘ über ein Produkt oder eine Marke eines Unternehmens lässt sich daraus keinesfalls ablesen. Wohl aber werden Attribute persuasiv explizit kommuniziert (quadratisch, praktisch, gut) oder impliziert (funktional, nützlich, ressourcenschonend, umweltbewusst, eigenständig, kreativ, individuell, überraschend) (zu Präsuppositionen vgl. bspw. Linke/Nussbaumer 2000), die der Leser als Angebot auch mit dem Produkt des Unternehmens assoziieren mag. Auf dieses implizite Angebot, das Fremdbild (Image) in Bezug zu setzen zu den kommunizierten oder vorausgesetzten Attributen, verweist der Text nicht ein einziges Mal (zu den grundlegenden Textfunktionen vgl. Rolf 2000 und Brinker 2010). Auf das dar- 
gestellte Selbstbild, das im Wesentlichen dieselben Attribute aufweist, wird nur ein einziges Mal im wiedergegebenen Slogan am Anfang des Textes metasprachlich verwiesen. In den Bildern ist das Produkt freilich präsent. Wie zu sehen sein wird, wirkt auch die Bildauswahl steuernd auf das sich anschließende Gespräch.

Das sich anbahnende Gespräch, zu dem mittels des Gewinnspiels eingeladen wird, hat das Thema der DIY-Hülle. Interessanterweise wird es nicht gekoppelt an die Gestaltung eigener Hüllen, sondern die bloße Antwort auf die gestellten Fragen „Wieso möchtet ihr die Hülle gewinnen und für was würdet ihr sie benutzen?“ Mit dieser Frage ist der Gesprächsverlauf nicht vollkommen offen, er wird auf der Basis von Erwartungserwartungen kanalisiert. Es eröffnet den Leser_innen auch ohne praktisches Nachahmen der Anleitung aktives Mitgestalten und Überraschen. Sie können verbal zeigen, dass sie kreativ und aktiv sind - und so werden sie in der Anrede „Hey kreative Schoko-Fans!“ des Artikels bereits gelobt und adressiert (vgl. Hartung 2001). Lob gilt als ein expressiver Gesprächsschritt ebenso wie Tadel und Dank oder der Ausdruck von Trauer und Freude: „Wir freuen uns riesig, wenn ihr die Hülle bastelt und uns ein Bild eurer genähten Hülle sendet.“ Das Gespräch hat neben einer informierenden Funktion (Anleitung) auch appellative Funktion (Aufforderung zum Mitmachen) und expressive Funktion (Etablierung und Stützung einer hier positiven Beziehung zwischen den Gesprächspartnern) (vgl. Heinemann 2000, Rolf 2000; Brinker/Sager 2006 und 2010).

Der Artikel wird mit insgesamt 134 Beträgen (Stand: 18.06.2014) kommentiert. Diese sollen jetzt hier nicht im Detail, sondern nur in Auswahl vorgestellt werden. Zwei Beiträge wurden beinahe identisch verdoppelt, die aus der Zählung ausgenommen werden. Von den dann 132 Kommentaren entfallen 100 Antworten auf Nutzer_ innen mit weiblichen Namen/Pseudonymen, 26 Antworten auf Nutzer_innen mit männlichen Namen/Pseudonymen und 6 Beiträge, bei denen der Name geschlechtsneutral gewählt wurde.

Die Kommentare lassen sich grob in drei Kategorien einordnen, nämlich zum einen in die, die auf die Frage „Wieso möchtet ihr die Hülle gewinnen und für was würdet ihr sie benutzen?“ tatsächlich antworten (responsiv). Sie machen quantitativ den größten Teil aus. Die Verwendungsmöglichkeiten nehmen zunächst die Vorschläge des Artikels auf (Hülle für „Smartphone“ bzw. „Handy“, „[Sonnen-]Brille“ oder „MP3-Player“ inkl. aller Äquivalente), daneben sollen die Hüllen aber auch als „Geschenk“, Aufbewahrungsmöglichkeit für „Schnuller“, „Stifte“, „Textmarker“, „Kugelschreiber“, „Kaugummi“, „Kleingeld“, „Führerschein“, „Perso[nalausweis]“, „Schlüssel“, „Kamera“, „Knabberzeugs“, „Notizzettel“, „Briefmarken“, „Büroklammern“, „Cremedose[n]“ usw., also all den „nervigen Kleinkram in der Handtasche“ als „fertige Tasche“ (Daniela) verwendet werden.

Eine andere Gruppe von Kommentaren (insgesamt 19) antwortet nur teilresponsiv, indem die DIY-Idee bewertet wird. Sie folgen also dem impliziten Appell des Instruktionstextes. Sie reichen damit von reiner Akklamation (,Das ist ja mal kreativ ... sieht toll aus“ [Daniela] bzw. „Coole Idee, und gar nicht so schwer nachzubasteln“ 
[Ramona]) bis zur Explikation des Recyclinggedankens („[D]as ist doch mal eine clevere Idee zur Vermeidung von Verpackungsmüll“ [volker]) oder der aus der DIYIdee resultierenden Einzigartigkeit („Damit hebt man sich von der Masse ab und zeigt, worauf man steht. Ein geniales Designprodukt.“ [Frank Krüger]).

Eine dritte Gruppe schließlich bezieht sich sowohl auf das Gewinnspiel als auch auf den impliziten Appell des Instruktionstextes bzw. die explizite Frage „Habt ihr noch weitere Ideen?“. Zwei Nutzer_innen wollen bspw. die Hüllen mit „Gurte[n]“ versehen, um sie an der „Seite/Bauch“ (Marco Bellersheim) oder über der „Schulter“ (Sarah) tragen zu können; nonresponsive Antworten sind nicht auszumachen.

In unserem Kontext könnte interessieren, wie die Antworten die Marke „Ritter Sport“ aufnehmen und wie diese Aufnahme in den Antworten repräsentiert ist. In insgesamt 27 Nennungen wird der Markenname in unterschiedlichen Varianten realisiert (,Ritter Sport“, „Rittersport“, „Ritter-Sport“ oder auch verkürzt in „Ritter-Hülle“), an das Corporate Wording indes hält sich freilich nur der Artikelautor Ben, der auf eine Nachfrage der Nutzerin Maria in Bezug auf die Gestaltung von weiteren Gewinnspielen antwortet („RITTER SPORT“). Daneben finden sich zahlreiche Wiederaufnahmen, die entweder spezielle Sorten erwähnen („Olympia“, „Knusperkeks“, „Marzipan“, „Karamell“, „Pfefferminz“ usw.) bzw. die Adjektive des Slogans zur attributiven Umschreibung aufgreifen („quadratische praktische Schokolade“ [Silke Frühling]) zwei Mal wird der Markenslogan eingearbeitet, wobei eine Antwort schon fast über das Ziel hinaus schießt: „Das nenn ich mal quadratisch - praktisch - gut! Auch wenn der süße Inhalt schon vernascht ist, bleibt es viereckig - praktisch - gut!“ (Carina).

Abschließend muss deutlich gesagt werden, dass sich aus den 132 Kommentaren kein Mehrpersonengespräch entwickelt, genauer sehen wir 129 Einzelgespräche vor uns, die mit dem Minimum von einem Sprecherwechsel und zwei Gesprächsschritten realisiert sind, aber wechselseitig sich gegenseitig anregen können (die Kommentare mit den „Gurten“ bspw. folgen direkt aufeinander, was wohl nicht nur als Koinzidenz aufzufassen ist). Allerdings entwickelt sich auch ein knappes Gespräch über vier Schritte und zwei Sprecherwechsel zwischen Maria und Ben (Blog-Autor) in Bezug auf die Art und Weise, wo und wie Gewinnspiele abgehalten werden können und sollen. Voraussetzung der folgenden Überlegungen ist, dass sich hinter dem (leider nicht sehr seltenen) Namen Maria zwei nicht identische Autor_innen verbergen, da nur eine ,der beiden“ ein Nutzerbild verwendet. Maria1 verfasst die erste Antwort auf Bens initiierenden Dialogakt, die vollresponsiv ist (13.06.2014, 14.25):

Mein Schatz ist auf Diät und das klappt eigentlich ganz gut - bis auf die Sache mit der Schokolade. Mit dieser Hülle als lustiges und kreatives Geschenk würde ich ihn wahnsinnig gern ein bisschen quälen...Gelb ist übrigens seine Lieblingsfarbe ;). (http://www.ritter-sport.de/ blog/2014/06/13/diy-ritter-sport-hulle-fur-das-smartphone-und-co-einfach-selbstgemacht/comment-page-1/\#comments, Zugriff am 17.12.2014)

Maria2 antwortet erstmals am 16.6.2014, 09.48, teilresponsiv auf Bens Eröffnung. Sie nimmt aber lediglich die Idee des Gewinnspiels auf, strukturell antwortet sie nicht mit 
einem Schritt eines erwartbaren Nachbarschaftspaares (wie Maria1), sondern mit einer Gegenfrage, die den Schwerpunkt auf ein anderes Thema legt („Anregung: [W]ieso macht man nicht alle Gewinnspiele von Ritter-Sport als Blog Gewinnspiele?[sic!]“). Sie steuert damit das Gespräch in eine andere Richtung: Trotz aller Steuerungsmechanismen kann man Gesprächsverläufe eben nicht vorhersehen. Der Frage folgt eine kurze Antwort Bens (in der zwei Schritte realisiert werden: Antwort und Verabschiedung) sowie eine kurzes Dankeschön seitens Maria2, was hier aber nicht weiter interessieren soll.

Wie sich an diesem kurzen Überblick über die Kommentare zum Artikel deutlich zeigt, arbeiten die Nutzer_innen mit ihren Antworten am Image der Marke und des Produkts mit, ohne dass dieses durch den Artikel selbst angestoßen würde - sie explizieren die im Artikel implizit vorausgesetzten Attribute praktisch, funktional, gut, nützlich, ressourcenschonend, umweltbewusst, eigenständig, kreativ, individuell, überraschend selbst. Sie leisten mit und durch und in Sprache Imagearbeit, sie arbeiten jeder für sich und doch gemeinsam in einem Prozess der Wissenskonstitution, -etablierung und -stabilisierung.

\section{Fazit und Ausblick}

Social Media beruht auf dem Prinzip der Dialogizität. Für eine erfolgreiche Unternehmenskommunikation, d. h. für eine, die die strukturellen Voraussetzungen der Formate des Web 2.0 akzeptiert, heißt das, dass sie sich von der Idee des ,instrumentellen Einsatzes‘ von ,unidirektionaler Kommunikation' verabschiedet haben muss. Social Media stellt verschiedene Kommunikationsräume zur Verfügung, wie den des Corporate Blogs, die strukturell nicht mehr als Präsentationsbühne oder ,Kanal‘ für die Darstellung von ,Informationen“ durch ein Unternehmen oder gar die Darstellung des unternehmerischen Selbstbildes (Identität) geeignet sind (Kap.1).

Die Corporate Blogs stellen Nutzer vor besondere Herausforderungen. Kennzeichnend für die Gespräche, die sich auf Corporate Blogs idealerweise entwickeln, ist, dass sie grundsätzlich eine starke Asymmetrie zwischen den Gesprächspartnern aufweisen. Diese Asymmetrie darf die Gespräche nicht dominieren, im Gegenteil. Vielmehr soll durch ein kollegiales Kommunikationsverhalten ,auf Augenhöhe‘ die strukturelle Asymmetrie als lediglich technisch bedingte ausgestellt werden. Da Gesprächsverläufe vor diesem Hintergrund ergebnisoffen und nicht vorhersagbar sind, man diese nur bedingt steuern und lenken kann und man in Gesprächen besser immer mit einer Antwort rechnet, ist man bei Gesprächsinitiierungen in Umgebungen des Web 2.0 dazu angehalten, solche Kommunikationsanlässe zu schaffen, die geeignet sind, Adressaten emotional auf der Beziehungsebene zu involvieren. Alle am Gespräch Beteiligten arbeiten gemeinsam am Gesprächssinn als kommunikativem Konstrukt, konstituieren, etablieren und stabilisieren damit Wissen durch Sprache 
(Kap. 2). Deshalb kann die Platzierung von ,Informationen` oder gar des Selbstbildes in einem initiierenden Beitrag nicht vorhersehbare Konsequenzen für das Fremdbild (Image) eines Produktes, eines Unternehmens usw. haben. Da das Gespräch im Kommunikationsraum Blog im Angesicht der Selbstpräsentation eines Produktes, einer Marke, eines Unternehmens usw. stattfindet, ist dies strukturell überhaupt nicht notwendig.

Nimmt man die unterschiedlichen Formen und Bedingungen von Social Media für kommunikationsstrategische Überlegungen ernst, dann kann, gemeinsam mit anderen, erfolgreich am Fremdbild (Image) und damit letztlich auch Selbstbild (Identät) gearbeitet werden, wie die exemplarische Analyse (Kap.3) oder auch der tendenziell ironische Kommentar von Purr Cat abschließend zeigen: „Wenn ich eine [sc. Hülle] gewinnen würde, würde ich Ritter Sport Minis reinpacken :D :D :D Erscheint mir als passende Aufbewahrung“.

\section{Literatur}

\subsection{Material}

„Audi Blog“ (http://blog.audi.de/(Zugriff am 04.07.2014). „Das Daimler-Blog“ (http://blog.daimler.de/(Zugriff am 04.07.2014). „Elbkind GmbH“ (http://elbkind.de/(Zugriff am 04.07.2014). „Ritter Sport“ (http://www.ritter-sport.de/(Zugriff am 04.07.2014). „Ritter Sport“-Blog (http://www.ritter-sport.de/blog (Zugriff am 04.07.2014). „DIY [sc. do it yourself]: RITTER SPORT Hülle für das Smartphone und Co. einfach selbstgemacht“ (http://www.ritter-sport.de/blog/2014/06/13/diy-ritter-sport-hulle-fur-das-smartphone-undco-einfach-selbstgemacht/(Zugriff am 04.07.2014).

\subsection{Sekundärliteratur}

Aerni, Markus/Manfred Bruhn (2008): Integrierte Kommunikation. Grundlagen mit zahlreichen Beispielen, Repetitionsfragen mit Lösungen und Glossar. Zürich.

Ainetter, Sylvia (2006): Blogs - literarische Aspekte eines neuen Mediums. Berlin.

Anders, Christina A./Markus Hundt/Alexander Lasch (2011): Der sprachliche Auftritt börsennotierter Unternehmen aus dem Energie- und Finanzdienstleistungssektor - Personalrekrutierung durch Sprache. Trends und Tendenzen in der sprachlichen Gestaltung von Karrierewebseiten (KIMATEK 2010).

Androutsopoulos, Jannis (2005): Online-Magazine \& Co. Publizistische Nischenangebote im Internet. In: Siever/Schlobinski/Runkehl, 98-131.

Androutsopoulos, Jannis (2007): Neue Medien - neue Schriftlichkeit?. In: Mitteilungen des Deutschen Germanistenverbandes 1/07, 72-97.

Androutsopoulos, Jannis (2014): Computer-mediated Communication and Linguistic Landscapes. In: Janet Holmes/Kirk Hazen (Hg.): Research Methods in Sociolinguistics. A Practical Guide. New Jersey, 74-90. 
Androutsopoulos, Jannis/Dirk Kasten (2005): Am Rande des Onlinejournalismus. In: Schütz u. a., 236-256.

Androutsopoulos, Jannis u. a. (Hg.) (2006): Neuere Entwicklungen in der linguistischen Internetforschung. Hildesheim u. a.

Barnes, Susan B. (2013): Social Networks. From Text to Video. New York.

Birkigt, Klaus/Marinus M. Stadler/Hans Joachim Funck (Hg.) (2002): Corporate Identity. GrundlagenFunktionen-Fallbeispiele. München.

Birkner, Karin/Janich, Nina (Hg.) (im Druck): Handbuch Text und Gespräch. Berlin/Boston.

Brinker, Klaus (2010): Linguistische Textanalyse. Eine Einführung in die Grundbegriffe und Methoden. 7. Aufl. Berlin.

Brinker, Klaus u. a. (Hg.) (2000): Text- und Gesprächslinguistik. Ein internationales Handbuch zeitgenössischer Forschung. 1. Halbbd. Berlin/New York.

Brinker, Klaus u. a. (Hg.) (2001): Text- und Gesprächslinguistik. Ein internationales Handbuch zeitgenössischer Forschung. 2. Halbbd. Berlin/New York.

Brinker, Klaus/Jörg Hagemann (2001): Gesprächssegmentierung nach Themen und Themenprogression. In: Brinker u. a. 2001, 1252-1263.

Brinker, Klaus/Sven F. Sager (2006): Linguistische Gesprächsanalyse. Eine Einführung. Berlin.

Bruhn, Manfred (2009): Marketing. Wiesbaden.

Bruhn, Manfred (2010): Kommunikationspolitik. Systematischer Einsatz der Kommunikation für Unternehmen. München.

Bruhn, Manfred (2011): Unternehmens- und Marketingkommunikation. Handbuch für integriertes Kommunikationsmanagement. 2., vollst. überarb. und erw. Aufl. München.

Bungarten, Theo (2005): Die Schwierigkeiten der Betriebswirtschaftslehre mit der Identität und der Kultur. In: Nina Janich (Hg.): Unternehmenskultur und Unternehmensidentität. Wirklichkeit und Konstruktion. Wiesbaden, 235-240.

Burghardt, Manuel u. a. (2013):Twitter als interaktive Erweiterung des Mediums Fernsehen: Inhaltliche Analyse von Tatort-Tweets. Darmstadt. In: http://gscl2013.ukp.informatik. tu-darmstadt.de/fileadmin/user_upload/Group_UKP/conferences/gscl2013/workshops/ Long-Paper-Tatort-Tweets_resubmittedVersion.pdf (Zugriff am 04.07.2014).

Crijns, Rogier/Janine Thalheim (Hg.) (2008): Kooperation und Effizienz in der Unternehmenskommunikation. Inner-und außerbetriebliche Kommunikationsaspekte von Corporate Identity und Interkulturalität. 2. Aufl. Wiesbaden.

Deppermann, Arnulf (2011): Konstruktionsgrammatik und Interaktionale Linguistik: Affinitäten, Komplementaritäten und Diskrepanzen. In: Alexander Lasch/Alexander Ziem (Hg.): Konstruktionsgrammatik III: Aktuelle Fragen und Lösungsansätze. Tübingen, 205-238.

Dörfel, Lars/Theresa Schulz (Hg.) (2011): Social Media in der Unternehmenskommunikation. Berlin. Dürscheid, Christa (2006): Merkmale der E-Mail-Kommunikation. In: Peter Schlobinski (Hg.): Von ${ }^{*}$ hdl $^{*}$ bis *cul8er*. Sprache und Kommunikation in den neuen Medien. Mannheim, 104-117.

Dürscheid, Christa (2009): E-Mail. Eine neue Kommunikationsform? In: Moraldo, 39-71.

Dürscheid, Christa/Arne Ziegler (2002): Kommunikationsform E-Mail. Tübingen.

Dürscheid, Christa/Sarah Brommer (2009): Getippte Dialoge in neuen Medien. Sprachkritische Aspekte und linguistische Analysen. In: Linguistik Online 37/1, 3-20.

Dürscheid, Christa/Carmen Frehner (2013): E-Mail communication. In: Herring/Stein/Virtanen, 35-54.

Dürscheid, Christa/Jan Georg Schneider (Hg.) (2015): Handbuch Satz, Äußerung, Schema. Berlin/ Boston.

Dürscheid, Christa/Karina Frick (2014): Keyboard-to-Screen-Kommunikation gestern und heute. SMS und WhatsApp im Vergleich. In: Mathias/Runkehl/Siever, 149-181. 
Dürscheid, Christa/Sarah Brommer (2013): Ist ein Freund noch ein Freund? Facebook und Sprachwandel. In: Der Deutschunterricht 2/2013, 28-40.

Ebert, Helmut/Klaus-Peter Konerding (2008): Sprachwandel, Organisationswandel, kultureller Wandel - Aspekte eines ganzheitlichen Modells für ein Kommunikations-Controlling. In: Crijns/ Thalheim (2008), 67-81.

Ehlich, Konrad/Claus Noack/Susanne Scheiter (1994): Instruktion durch Text und Diskurs. Wiesbaden.

Eisenlauer, Volker (2013): A critical hypertext analysis of Social Media. The true colours of Facebook. London.

Esch, Franz-Rudolf/Andreas Hermann/Henrik Sattler (2008): Marketing. Eine managementorientierte Einführung. München.

Fishman, Joshua A. 1972. Language in Sociocultural Change. Stanford.

Förster, Hans-Peter (1994): Corporate Wording. Konzepte für eine unternehmerische Schreibkultur. Frankfurt/New York.

Fraas, Claudia/Achim Barczok (2006): Intermedialität - Transmedialität. Weblogs im öffentlichen Diskurs. In: Androutsopoulos u. a., 132-160.

Frick, Karina (2014): Liebeskommunikation über Facebook. Eine korpusbasierte Untersuchung kommunikationstheoretischer und sprachlicher Merkmale der Paar-Kommunikation auf Facebook. In: Networx 65, http://www.mediensprache.net/networx/networx-65.pdf (Zugriff am 16.12.2014).

Gioia, Dennis A. u. a. (2000): Organizational identity, image, and adaptive instability. In: The Academy of Management Review 25/1, 63-81.

Goffman, Erving (1996): Interaktionsrituale. Über Verhalten in direkter Kommunikation. Frankfurt a. M.

Gruber, Helmut (2001): Die Struktur von Gesprächssequenzen. In: Brinker u. a. 2001, 1226-1240.

Gülich, Elisabeth/Heiko Hausendorf (2000): Vertextungsmuster Narration. In: Brinker u. a. 2000, 369-384.

Handler, Peter (2006): Zur Narrativik des digital storytelling. Textkonstitution in sequenziell angelegten Web-Formaten. In: Androutsopoulos u. a., 209-234.

Hartung, Martin (2001): Formen der Adressiertheit der Rede. In: Brinker u. a. 2001, 1348-1355.

Heidegger, Gerald (2004): Vom Pamphlet zum Blog. In: Praesent 3, 45-50.

Heinemann, Margot (2000): Textsorten des Alltags. In: Brinker u. a. 2000, 604-613.

Herring, Susan C./Dieter Stein/Tuija Virtanen (Hg.) (2013). Pragmatics of Computer-Mediated Communication. Berlin/Boston.

Hess-Lütich, Ernest W. B. (2007): Netzliteratur - ein neues Genre?. In: Michael Stolz/Lucas Marco Gisi/Jan Loop (Hg.): Literatur und Literaturwissenschaft auf dem Weg zu den neuen Medien. Eine Standortbestimmung. Zürich, 225-43.

Hindelang, Götz (2010): Einführung in die Sprechakttheorie. Sprechakte, Äußerungsformen, Sprechaktsequenzen. 5. Aufl. Berlin/Boston.

Hohm, Hans-Jürgen (2006): Soziale Systeme, Kommunikation, Mensch. Eine Einführung in soziologische Systemtheorie. Weinheim.

Holly, Werner (2001): Beziehungsmanagement und Imagearbeit. In: Brinker u. a. 2001, 1382-1393.

Hundt, Markus (2009): Verhaltensregulierung und Identitätsstiftung durch Unternehmensverfassungen. Corporate Governance unter sprachlichen Aspekten. In: Ekkehard Felder/Marcus Müller (Hg.): Wissen durch Sprache. Theorie, Praxis und Erkenntnisinteresse des Forschungsnetzwerkes „Sprache und Wissen“. Berlin/New York, 479-502.

Kastens, Inga E. (2008): Linguistische Markenführung: die Sprache der Marken - Aufbau, Umsetzung und Wirkungspotenziale eines handlungsorientierten Markenführungsansatzes. Münster. 
Kindt, Walther (2001): Konventionen, Regeln und Maximen in Gesprächen. In: Brinker u. a. 2001, $1178-1187$.

Koch, Peter/Wulf Oesterreicher (1985): Sprache der Nähe - Sprache der Distanz. Mündlichkeit und Schriftlichkeit im Spannungsfeld von Sprachtheorie und Sprachgeschichte. In: Romanistisches Jahrbuch, 36. Berlin/New York, 15-43.

Koch, Peter/Wulf Oesterreicher (1994): Funktionale Aspekte der Schriftkultur. Functional Aspects of Literacy. In: Hartmut Günther/Otto Ludwig (Hg.): Schrift und Schriftlichkeit. Ein interdisziplinäres Handbuch internationaler Forschung. 1. Halbbd.. Berlin/New York, 587-604.

Krallmann, Dieter/Andreas Ziemann (2001): Grundkurs Kommunikationswissenschaft. Mit einem Hypertext-Vertiefungsprogramm im Internet. München.

Kreutzer, Ralf T. (2012): Praxisorientiertes Online-Marketing. Konzepte - Instrumente - Checklisten. Wiesbaden.

Lakoff, George/Elisabeth Wehling (2009): Auf leisen Sohlen ins Gehirn. Politische Sprache und ihre heimliche Macht. 2., aktual. Aufl. Heidelberg.

Linke, Angelika/Markus Nussbaumer (2000): Konzepte des Impliziten: Präsuppositionen und Implikaturen. In: Brinker u. a. 2000, 435-448.

Marx, Konstanze/Georg Weidacher (Hg.) (2014). Internetlinguistik. Ein Lehr- und Arbeitsbuch. Tübingen.

Mast, Claudia (2010): Unternehmenskommunikation. 4. Aufl. Stuttgart.

Mathias, Alexa/Jens Runkehl/Torsten Siever (Hg.) (2014): Sprachen? Vielfalt! Sprache und Kommunikation in der Gesellschaft und den Medien. Eine Online-Festschrift zum Jubiläum von Peter Schlobinski. networx 64, http://www.mediensprache.net/de/networx/networx-64.aspx (Zugriff am 16.12.2014).

Möhn, Dieter (1991): Instruktionstexte. Ein Problemfall bei der Textidentifikation. In: Klaus Brinker (Hg.): Aspekte der Textlinguistik. Hildesheim, 183-212.

Moraldo, Sandro (Hg.) (2009): Internetkommunikation. Neue Sprach- und Kommunikationsformen im World Wide Web. Bd.1: Kommunikationsplattformen. Rom.

Motsch, Wolfgang (2000): Handlungsstrukturen von Texten. In: Brinker u. a. 2000, 414-421.

Müller, Klaus (2001): Probleme der Sinnkonstituierung in Gesprächen. In: Brinker u. a. 2001, 1196-1212.

Piwinger, Manfred/Ansgar Zerfaß (Hgg.) (2007): Handbuch Unternehmenskommunikation. Wiesbaden.

Porombka, Stephan (2012): Duden. Schreiben unter Strom. Experimentieren mit Twitter, Blogs, Facebook \& Co. Mannheim.

Puschmann, Cornlius (2010): The corporate blog as an emerging genre of computer-mediated communication. Features, constraints, discourse situation. Göttingen.

Rath, Rainer (2001): Gesprächsschnitt und Höreraktivitäten. In: Brinker u. a. 2001, 1213-1226.

Rolf, Eckard (1992): Die Funktionen der Gebrauchstextsorten. Berlin/New York.

Rolf, Eckard (2000): Textuelle Grundfunktionen. In: Brinker u. a. 2000, 422-434.

Rolf, Eckard/Jörg Hagemann (2001): Die Bedeutung der Sprechakttheorie für die Gesprächsforschung. In: Brinker u. a. 2001, 885-896.

Runkehl, Jens/Peter Schlobinski/Torsten Siever (Hg.) (2013): Sprache - Medien - Innovationen. Bd 4. Frankfurt a. M.

Salzer, Eva (2011): Quintessenz der Unternehmenskommunikation. Wie Sie Ihre Ziele im Dialog mit Ihrem Stakeholder besser erreichen können. Berlin/Heidelberg.

Schlobinski, Peter/Torsten Siever (2000): Kommunikationspraxen im Internet. In: Der Deutschunterricht 1/2000, 54-65. 
Schmid, Beat F./Boris Lyczek (2008): Die Rolle der Kommunikation in der Wertschöpfung der Unternehmung. In: Beat F. Schmid/Boris Lyczek (Hg.): Unternehmenskommunikation. Kommunikationsmanagement aus Sicht der Unternehmensführung. Wiesbaden, 3-150.

Schulz von Thun, Friedemann (1981): Miteinander reden. Störungen und Klärungen. Psychologie der zwischenmenschlichen Kommunikation. Reinbek bei Hamburg.

Schulz von Thun, Friedemann (o. Datum): Das Kommunikationsquadrat. Online unter URL: http:// www.schulz-von-thun.de/index.php?article_id=71 (Zugriff am: 23.03.2015).

Schuster, Jörg (2013): Kreativer Austausch oder digitaler Masochismus?. In: Literaturkritik.de 15/4, 31-35.

Schütz, Astrid u. a. (Hg.) (2005): Neue Medien im Alltag. Befunde aus den Bereichen Arbeit, Lernen und Freizeit. Göttingen.

Schwender, Clemens (Hg.) (1999): Zur Geschichte der Gebrauchsanleitung. Theorien - Methoden Fakten. Frankfurt.

Schwitalla, Johannes (1976): Dialogsteuerung. Vorschläge zur Untersuchung. In: Franz Josef Berens u. a. (Hg.). Projekt Dialogstrukturen. Ein Arbeitsbericht. Mit einer Einleitung von Hugo Steger. Ismaning, 73-97.

Schwitalla, Johannes (2001): Gesprochene-Sprache-Forschung und ihre Entwicklung zu einer Gesprächsanalyse. In: Brinker u. a. 2001, 897-903.

Seidler, Detlev (1997): Unternehmenskultur und Corporate Identity. Ihre Ansätze in der Wirtschaftslinguistik. In: Theo Bungarten (Hg.): Aspekte der Unternehmungskultur und Unternehmensidentität in der historischen Wirtschaftslinguistik. Tostedt, 91-107.

Siever, Torsten (2013a): Microblogs global. Eine internationale Studie zu Twitter \& Co. aus der Perspektive von zehn Sprachen und elf Ländern. In: Runkehl/Schlobinski/Siever, 41-74.

Siever, Torsten (2013b): Zugänglichkeitsaspekte zur Kommunikation im technischen Zeitalter. In: Konstanze Marx/Monika Schwarz-Friesel (Hg.): Sprache und Kommunikation im technischen Zeitalter. Wieviel Internet (v)erträgt unsere Gesellschaft?. Berlin/Boston, 7-25.

Siever, Torsten (2014a): Digitale Welt: Kommunikative Folgen und Folgen der Kommunikation. In: Mathias/Runkehl/Siever, 197-234.

Siever, Torsten (2014b): Grammatik in digitalen Kommunikationsformen. In: Der Deutschunterricht 3/2014, 49-61.

Siever, Torsten/Peter Schlobinski/Jens Runkehl (Hg.) (2005): Websprache.net. Berlin.

Tiittula, Liisa (2001). Formen der Gesprächssteuerung. In: Brinker u. a. 2001, 1361-1374.

Zerfaß, Ansgar (2005): Corporate Blogs: Einsatzmöglichkeiten und Herausforderungen. In: BIG Bloginitiative Germany: 27.01.2005. http://www.albatros-media.at/pdfs/ CorporateBlogs-AZ-270105.pdf (Zugriff am 16.12.2014).

Zintzen, Christiane (2012): Blogliteratur: Medium oder Message? Am Beispiel der Plattform litblogs. net - Literarische Weblogs in deutscher Sprache. In: Meri Disoki/Ursula Klingenböck/Stefan Krammer (Hg.): (Ver)Führungen. Räume der Literaturvermittlung. Innsbruck u. a., 84-104. 